\title{
Accuracy of QT Measurement in the EASI-derived 12-lead ECG
}

\author{
Juan Pablo Martínez, Pablo Laguna, Salvador Olmos, Olle Pahlm, Jonas Petersson and Leif Sörnmo
}

\begin{abstract}
The purpose of this study is to assess QT interval measurements from the EASI 12-lead ECG as compared to the standard 12-lead ECG. The QT interval was automatically measured in simultaneously recorded standard and EASI 12lead ECGs, using a validated wavelet-based delineator. The agreement between the two sets of measurements was quantified both on a lead-by-lead basis and a multilead basis. The results show an acceptable agreement between QT measurements in the two lead systems, with correlation coefficients (CC) 0.91-0.98 depending on the lead. Standard deviations range from $9.2 \mathrm{~ms}$ to $26.4 \mathrm{~ms}$ depending on the selected lead. In a few patients large inter-system differences were found, mainly due to different $T$ wave morphologies. Using a multilead delineation, QT intervals were considerably more stable than single-lead measurements and resulted in a much better agreement between the two lead systems (CC: 0.98, QT difference: $1.1 \mathrm{~ms} \pm 9.8 \mathrm{~ms})$. Thus, EASI-derived 12lead ECG may be used for reliable QT monitoring when the multilead delineation approach is adopted.
\end{abstract}

\section{INTRODUCTION}

$\mathrm{I}_{3}^{\mathrm{T}}$ $\mathrm{T}$ is well-known that prolonged cardiac repolarization is associated with susceptibility to ventricular tachyarrhythmias, usually in the form of Torsade de Pointes, that can degenerate into life-threatening arrhythmias such as ventricular fibrillation. This phenomenon is manifested in the electrocardiogram (ECG) as a prolongation of the QT interval. Despite its limitations [1], the QT interval remains the most widely used index for assessing the propensity to ventricular arrhythmias.

Certain drugs have the ability to delay myocardial repolarization [2]. This fact has resulted in a substantial number of regulatory actions, including withdrawals of potential cardiotoxic drugs from the market. Drug regulatory agencies worldwide have adopted the ICH E14 guidelines, which require rigorous monitoring of the QT interval [3].

In the last 2 decades an alternative electrode placement system, the EASI lead system, has been introduced [4]. EASI lead monitoring is based on only 5 electrodes, one of which is a ground electrode. The 4 active electrodes are positioned as follows: 2 are placed on the sternum and 2 in the right and left midaxillary lines (Fig. 1). Based on the

This work was supported in part by projects TEC-2004-05263-C02 from MCYT/FEDIR, PI04/0689 from FIS (Spain), and by Philips Corp.

J. P. Martínez, S. Olmos and P. Laguna are with the Communications Technology Group, Aragon Institute of Engineering Research, University of Zaragoza, Aragon, Spain. (e-mail: \{jpmart,olmos,laguna\}@unizar.es).

O. Pahlm is with the Dept of Clinical Physiology, Lund University Hospital. Sweden.

J. Petersson is with Experimental Medicine, AstraZeneca R\&D. Sweden.

L. Sörnmo is with Department of Electroscience. Lund Institute of Technology. Sweden. signals from these positions it is possible to mathematically construct an "EASI 12-lead ECG". The use of EASI, requiring less electrodes, would facilitate continuous monitoring in phase I clinical trials.

Several papers have described the agreement between the EASI and the standard 12-lead ECG regarding diagnostic accuracy [5-7], myocardial ischemia and infarction classification [8] and waveform measurements [9].

The purpose of the present study is to assess QT interval measurements from the EASI 12-lead ECG as compared to the standard 12-lead ECG; an issue which has not previously been addressed in the literature.

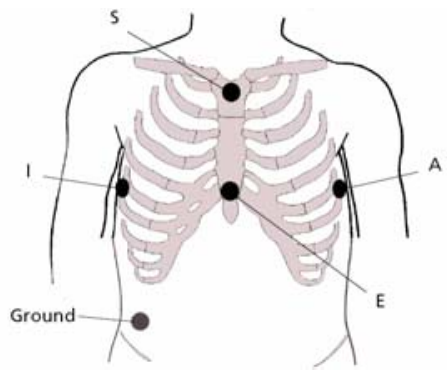

Fig. 1: Locations of the active electrodes (E. A. S. I).

\section{MATERIALS AND METHODS}

\section{A. Study population and acquisition}

The initial study group consisted of 200 patients from Rui Jin Hospital, Taiwan, having a wide range of ages and cardiac conditions. For each patient, the standard 12-lead and EASI ECGs were recorded simultaneously during 11 seconds, using a Philips resting ECG recorder (sampling rate $500 \mathrm{~Hz}$, amplitude resolution 5 microvolts).

The exclusion criteria for ECG recordings were pacemaker, atrial fibrillation/flutter, right/left bundle branch block, WPW syndrome, QRS duration $>120 \mathrm{~ms}$, ST elevation acute myocardial infarction, resting heart rate $>100$ or $<50$ beats/min, bad electrode location, or insufficient technical quality of the ECG recording. Following exclusion, a total of 104 patients were used for further evaluation.

\section{B. Single-lead waveform delineation}

Automatic waveform delineation of the heartbeat was performed with a multiscale wavelet-based delineator previously described and validated [10]. The onsets, peaks and ends of the ECG waves were determined using the differentiated ECG, smoothed at different scales, which was obtained by means of the discrete wavelet transform, using a quadratic spline wavelet. 
Using an annotated QT database [11], disagreement between expert annotations and automatic delineation was found to be comparable to interexpert differences in the same database [10].

\section{EASI vs standard leads QT comparison}

The EASI 12-lead ECG was derived using the transformation matrix given by Feild et al. [12]. QT intervals were automatically measured in the two sets of simultaneously recorded ECGs. For a given patient, indexed by $p=\{1, \ldots, 104\}$, and beat, indexed by $n=\left\{1, \ldots, N_{p}\right\}$, the method supplied up to 24 measurements defining QRS onset, $\mathrm{T}$ wave end, and related QT interval, denoted by $Q_{o n}^{s}{ }_{l, p}$, Tend ${ }_{l, p}^{s}$, and $Q T_{l, p}^{s}$, respectively. The leads are indexed by $\boldsymbol{l}=\left\{\mathrm{V}_{1}, \mathrm{~V}_{2}, \mathrm{~V}_{3}, \mathrm{~V}_{4}, \mathrm{~V}_{5}, \mathrm{~V}_{6}, \mathrm{I}\right.$, II, III, aVL, aVR, $\mathrm{aVF}\}$, and the lead system by $s=\{$ EASI, STD $\}$. For each lead, differences between measurements of the derived and standard leads were computed and denoted

$$
\begin{gathered}
\Delta \text { Qon }_{l, p}=\text { Qon }_{l, p}^{E A S I}-\text { Qon }_{l, p}^{S T D}, \\
\Delta \text { Tend }_{l, p}=\text { Tend }_{l, p}^{E A S I}-\text { Tend }_{l, p}^{S T D}, \\
\Delta Q T_{l, p}=Q T_{l, p}^{E A S I}-Q T_{l, p}^{S T D},
\end{gathered}
$$

After discarding the first and last detected beats, the mean QT interval was computed in every patient for each lead and lead system and denoted $Q T_{l, p}^{E A S I}$ and $Q T_{l, p}^{S T D}$. The mean differences in the measurements of QRS onset, $\mathrm{T}$ wave end, and QT interval between the derived and standard leads for a given patient and lead are denoted by $\Delta$ Oon $_{l, p} \Delta$ Tend $_{l, p}$ and $\Delta Q T_{l, p}$.

To avoid unstable QT measurements, leads with less than 5 detected $\mathrm{T}$ waves or with large QT variability (the intralead standard deviation exceeding $30 \mathrm{~ms}$ ) in any lead configuration were discarded from further analysis. The main reasons for instability were the presence of lowamplitude/flat $\mathrm{T}$ waves and artifacts.

\section{Multilead waveform delineation}

Lead-by-lead comparison between the EASI and standard ECG can be considerably influenced by small differences in axis orientation of the lead systems. It is therefore desirable to consider a multilead approach in which all 12 leads are taken into account to produce a global QRS onset and T wave end for each beat. Physiologically, the QT interval is ideally defined as the difference between the latest $T$ wave end and the earliest QRS onset among the 12 leads. However, this strategy is extremely sensitive to delineation and classification errors $[1,13]$.

To reduce the risk of such errors, a multilead rule was employed for the determination of a global QRS onset and T wave end [13]. First, the QRS onsets and T wave ends of the different leads are sorted (a maximum of 12 for each of the two). Then, the global QRS onset is defined as the earliest QRS onset followed by at least 3 other onsets in the next $\delta$ ms. In the same way, the last $\mathrm{T}$ wave end preceded by at least 3 other ends in the previous $\delta \mathrm{ms}$ is considered to be the global $\mathrm{T}$ wave end. The value of $\delta$ was set to $12 \mathrm{~ms}$, previously applied in [10] for validation of the above wavelet-based delineator on the CSE multilead database [14]. This multilead rule, being robust to delineation errors, preserves the physiological meaning of the QT interval as the period from the beginning of ventricular depolarization to the end of ventricular repolarization.

\section{RESULTS}

Table 1 presents the mean values of $\Delta Q T_{l, p}$ across patients, i.e., $\overline{\Delta Q T}_{l}=\frac{1}{P_{l}} \sum_{p=1}^{P_{l}} \Delta Q T_{l, p}$, and the similarly defined $\overline{\Delta Q o n}_{l}$ and $\overline{\Delta T e n d}_{l}$ (mean \pm standard deviation). For each lead, the number of patients $\left(P_{l}\right)$ with stable QT measurements is presented.

The Pearson correlation coefficients between single-lead TABLE I

MEASUREMENT DIFFERENCES BETWEEN STANDARD AND EASI LEADS.

\begin{tabular}{ccccc}
\hline Lead & $\begin{array}{c}\text { \# patients } \\
P_{l}\end{array}$ & $\begin{array}{c}\overline{\Delta \boldsymbol{Q T}}_{\boldsymbol{l}} \\
(\mathrm{ms})\end{array}$ & $\begin{array}{c}\overline{\Delta \boldsymbol{Q o n}}_{\boldsymbol{l}} \\
(\mathrm{ms})\end{array}$ & $\begin{array}{c}\overline{\Delta \boldsymbol{T e n d}}_{\boldsymbol{l}} \\
(\mathrm{ms})\end{array}$ \\
\hline $\mathrm{V}_{1}$ & 76 & $0.9 \pm 25.5$ & $-1.0 \pm 8.6$ & $0.0 \pm 21.1$ \\
$\mathrm{~V}_{2}$ & 81 & $-1.0 \pm 20.8$ & $1.0 \pm 7.5$ & $0.0 \pm 18.8$ \\
$\mathrm{~V}_{3}$ & 79 & $2.7 \pm 17.9$ & $-1.0 \pm 4.8$ & $1.7 \pm 17.5$ \\
$\mathrm{~V}_{4}$ & 79 & $-0.8 \pm 16.4$ & $0.2 \pm 4.2$ & $-0.7 \pm 16.5$ \\
$\mathrm{~V}_{5}$ & 88 & $-3.5 \pm 13.6$ & $-0.2 \pm 4.6$ & $-3.7 \pm 12.8$ \\
$\mathrm{~V}_{6}$ & 87 & $-0.1 \pm 12.5$ & $-0.3 \pm 6.3$ & $-0.4 \pm 9.4$ \\
\hline aVL & 67 & $-0.8 \pm 20.1$ & $2.3 \pm 8.3$ & $1.5 \pm 18.6$ \\
I & 74 & $-1.7 \pm 9.2$ & $2.8 \pm 5.4$ & $1.1 \pm 7.7$ \\
aVR & 80 & $1.0 \pm 12.8$ & $0.8 \pm 7.2$ & $1.8 \pm 10.1$ \\
II & 84 & $-1.1 \pm 19.1$ & $-0.3 \pm 8.5$ & $-1.5 \pm 14.7$ \\
aVF & 82 & $-0.2 \pm 21.8$ & $-0.9 \pm 10.5$ & $-1.0 \pm 20.9$ \\
III & 76 & $-0.4 \pm 26.4$ & $-0.5 \pm 9.9$ & $-1.0 \pm 24.8$ \\
\hline Multilead & 98 & $1.1 \pm 9.8$ & $0.5 \pm 3.6$ & $1.6 \pm 9.4$ \\
\hline
\end{tabular}

QT intervals measured in both lead systems are as follows: (V1: 0.90, V2: 0.94, V3: 0.64, V4: 0.96, V5: 0.97, V6: 0.97, aVL: 0.91, I: 0.98, aVR: 0.97, II: 0.93, aVF: 0.91, III: 0.88). The correlation coefficient between multilead QT intervals was 0.98 .

Figure 2 shows, the Bland-Altman plots for both measurements in lead II (commonly used for single-lead QT measurement) as well as with the multilead measurement.

To gain insight into the error distribution, the cumulative distributions of the absolute QT differences between STD and EASI leads $\left|\Delta Q T_{l, p}\right|$ are shown in Fig. 3.

Most of the largest QT measurement differences were caused by differences in $\mathrm{T}$ wave morphology between the two lead systems. An example with large QT differences is shown in Figs. 4 and 5. It is obvious that the change in $T$ wave morphology of the derived leads V1 and V2 causes large difference in QT measurements. Fig. 5 illustrates the 

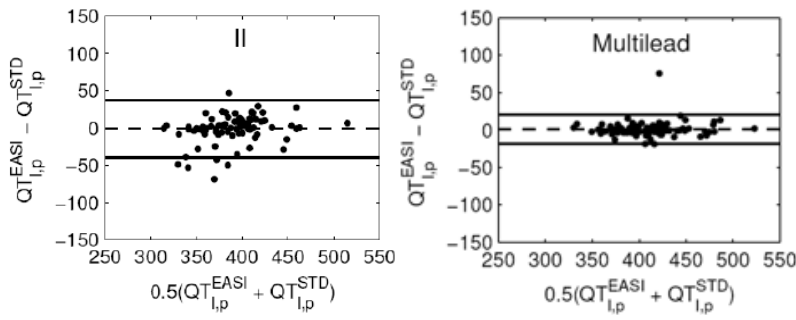

Fig. 2. Bland-Altman plot for the QT multilead measurements in EASI-derived and standard leads. Each point represents the agreement between both measurments in one patient.

ability of the multilead approach to measure QT interval robust with respect to changes in the electrical axis.

\section{DISCUSSION}

\section{A. Single-lead QT measurements.}

Table 1 shows that QT intervals measured in EASIderived leads differ, in average, less than one sampling interval $(2 \mathrm{~ms})$ from measurements in the standard leads, except in V3, V5 (with mean differences of $2.7 \mathrm{~ms}$ and -3.5 $\mathrm{ms}$, respectively). The standard deviation of the differences is strongly dependent on the selected lead; the most stable measurements were found in V5 $(13.6 \mathrm{~ms})$, V6 $(12.5 \mathrm{~ms})$, I $(9.1 \mathrm{~ms})$, and $-\mathrm{aVR}(12.8 \mathrm{~ms})$, all fulfilling the multilead tolerances proposed in [14]. Note that all these leads have an important component in the "right-to-left" direction, i.e., the direction of the $\mathrm{X}$ axis as defined in vectorcardiography. On the other hand, the largest standard deviations across patients occurred in III $(26.4 \mathrm{~ms})$ and V1 $(25.5 \mathrm{~ms})$, most likely explained by the fact that these leads are more influenced by axis deviations.

The variability in QT interval measurements is mainly due to the variability in the $T$ wave end delineation as the differences are much smaller for the QRS onset than for the $\mathrm{T}$ wave end. The mean differences in the QRS onset determination remained bounded by $1 \mathrm{~ms}$, except for leads $\mathrm{aVL}$ and I (Table 1) in which a bias of more than one sample interval was found. The standard deviation was lower than $10.5 \mathrm{~ms}$ in all leads. Differences were larger in the $\mathrm{T}$ wave end delineation: the largest bias was found in

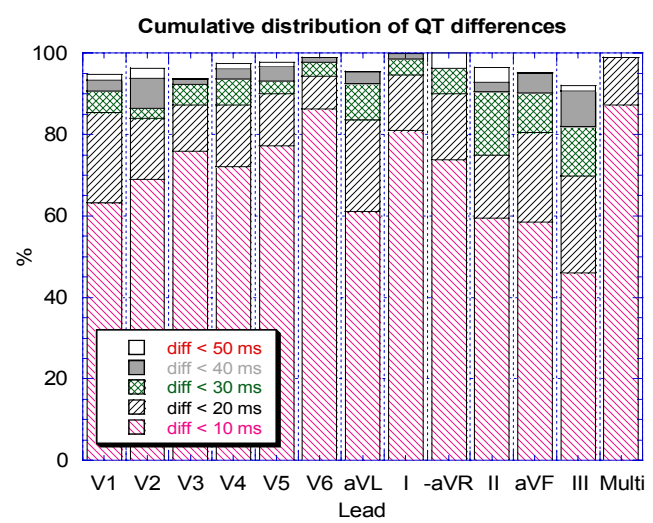

Fig. 3. Cumulative distribution of absolute QT differences.

leads V5 (-3.6 ms) and aVR (2.6 ms), while the bias was lower than the sampling interval in the other leads. The standard deviation of the differences ranged from $7.7 \mathrm{~ms}$ (I) to 24.8 (III). The pattern of the lead-by-lead differences in T wave end measure was similar to that of the QT interval.

The largest correlation between measurements in both

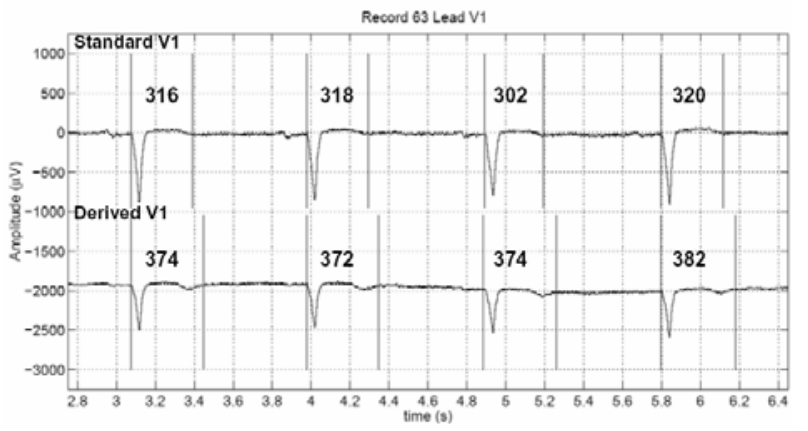

(a)

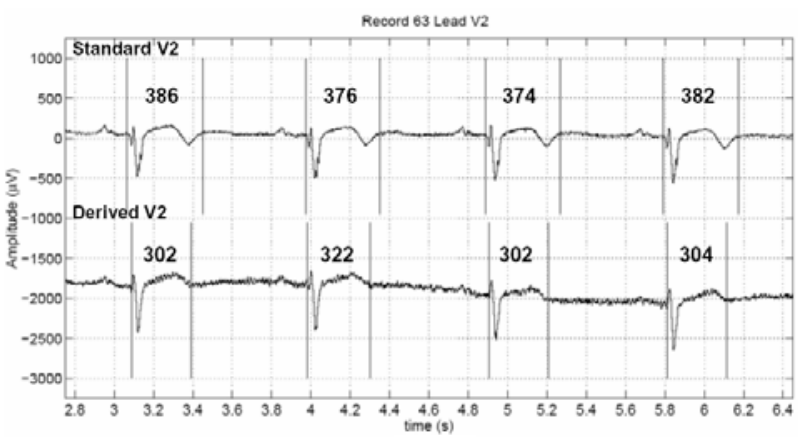

(b)

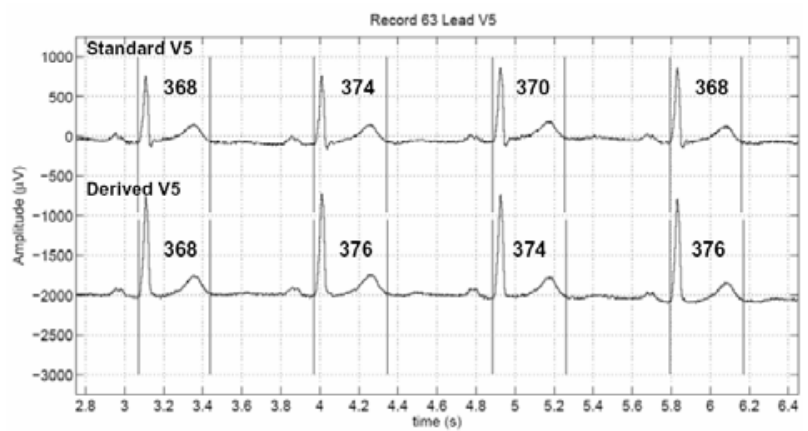

(c)

Fig. 4. Comparison of QT measurements in standard and EASIderived V1, V2 and V5, for a patient with large differences.

systems was also found in leads V5, V6, I and -aVR ( $\mathrm{r}>$ 0.97 in all of them), whereas the lowest correlation was found in leads V1 ( $\mathrm{r}=0.9)$ and III $(\mathrm{r}=0.88)$. The BlandAltman plots did not show any clear trend in the intermeasurement differences.

The distributions of the QT differences were very dependent on the selected lead. While certain leads such as V6 or I exhibited QT differences lower than $10 \mathrm{~ms}$ in more than $80 \%$ of the records, more than half of the records exhibited differences larger than $10 \mathrm{~ms}$ in other leads such as III (Fig. 3). As illustrated by the example in Fig. 4 and 5, the largest differences were mainly due to changes in $T$ wave morphology between the two lead systems. 


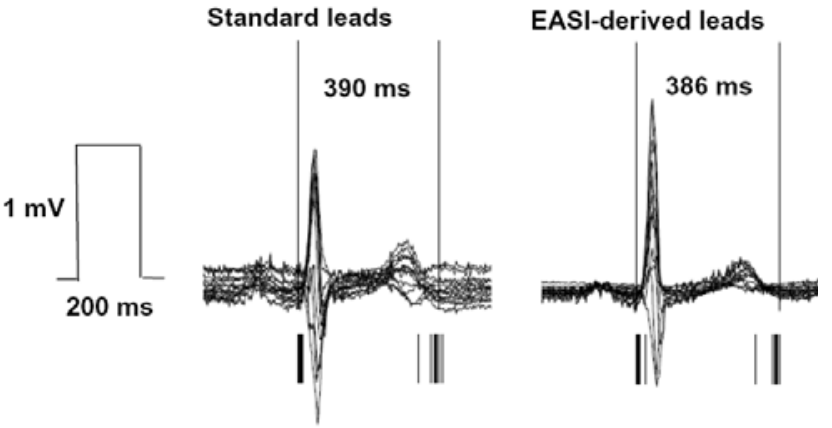

Fig. 5. Multilead delineation for the signal displayed in Figure 6, but now displayed with a superimposed format. The global QRS onset and $\mathrm{T}$ wave end are marked by long lines and single-lead delineations with short lines.

Since no manual annotations were available, it is not evident which part of the inter-systems variability should be attributed to delineator accuracy. However, as the waveletbased delineator has been validated on well-known test databases, we can conclude that the inter-system variability, in most leads, was equal or lower than the standard deviation of the differences with manual annotations obtained from other databases.

Regarding $\mathrm{T}$ wave detection, $\mathrm{T}$ waves were delineated in more than $95 \%$ of the beats for either standard or EASIderived leads. Assuming the standard lead as the gold standard for $\mathrm{T}$ wave existence, the best sensitivity ( $>99 \%$ ) was attained in leads V5, V6, I, and aVR.

\section{B. Multilead QT measurements.}

The multilead approach used in this work reduces intersystem differences in QT measurements, obtaining a negligible bias (1.1 ms for QT, $0.5 \mathrm{~ms}$ for QRS onset, and $1.6 \mathrm{~ms}$ for $\mathrm{T}$ wave end) and standard deviations comparable to those of the best lead measurements $(9.8 \mathrm{~ms}$ for QT interval, 3.6 ms for QRS onset and 9.4 $\mathrm{ms}$ for $\mathrm{T}$ wave end). Moreover, stable beat-to-beat QT measurements are obtained in 98 out of the 104 records. These inter-system differences are negligible in view of the intrinsic variability of the multilead delineator observed in the CSE database [10].

Multilead delineation also attained the maximum correlation between both systems $(\mathrm{r}=0.98)$, with differences shorter than $10 \mathrm{~ms}$ in almost $90 \%$ of the records and shorter than $20 \mathrm{~ms}$ in all except one record (Fig. 3).

The adopted multilead approach offers a stable QT measurement with negligible differences between the two lead systems, despite the fact that it ultimately relies on single lead measurements. A delineation approach based on the VCG loop, such as the one proposed in [15], may offer measurements which are even less dependent on the selected lead system.

\section{CONCLUSiOnS}

Differences in QT measurements in standard and EASIderived leads are strongly dependent on the selected lead. In particular, leads with a large X-component exhibited the lowest differences. Nonetheless, measurement differences were always within the standard tolerances given by the CSE working party (e.g. $30.6 \mathrm{~ms}$ for the $\mathrm{T}$ wave end).

However, we found quite high differences in several records. This is quite relevant, since a small number of outlier measurements can bias the conclusion of a study on the QT prolongation. The largest differences were due to changes in $\mathrm{T}$ wave morphology, which can be attributed to differences in projection due to changes in the direction of the corresponding lead vectors. This hypothesis is reinforced by the better agreement obtained when using a multilead approach.

\section{REFERENCES}

[1] Malik M. Errors and misconceptions in ECG measurement used for the detection of drug induced QT interval prolongation. J. Electrocardiol. 2004; 37(suppl):25-33.

[2] Yap YG, Camm AJ. Drug induced QT prolongation and torsades de pointes. Heart. 2003 Nov;89(11):1363-72.

[3] FDA. Guidance for Industry. E14 Clinical Evaluation of QT/QTc Interval Prolongation and Proarrhythmic Potential for NonAntiarrhythmic Drugs. October 2005. http://www.fda.gov/cder/guidance/6922fnl.pdf (accessed: Nov-2005).

[4] Dower GE, Yakush A, Nazzal SB, Jutzy RV, Ruiz CE. Deriving the 12-lead electrocardiogram from four (EASI) electrodes. J Electrocardiol. 1988;21 Suppl:S182-7.

[5] Drew BJ, Adams MG, Pelter MM, Wung SF, Caldwell MA. Comparison of standard and derived 12-lead electrocardiograms for diagnosis of coronary angioplasty-induced myocardial ischemia. Am J Cardiol. 1997 Mar 1;79(5):639-44.

[6] Drew BJ, Pelter MM, Wung SF, Adams MG, Taylor C, Evans Jr GT, Foster E. Accuracy of the EASI 12-lead electrocardiogram compared to the standard 12-lead electrocardiogram for diagnosing multiple cardiac abnormalities. J Electrocardiol. 1999;32 Suppl:38-47

[7] Horacek BM, Warren JW, Stovicek P, Feldman CL. Diagnostic accuracy of derived versus standard 12-lead electrocardiograms. J Electrocardiol. 2000;33 Suppl:155-60.

[8] Rautaharju PM, Zhou SH, Hancock EW et al. Comparability of 12lead ECGs derived from EASI leads with standard 12-lead ECGS in the classification of acute myocardial ischemia and old myocardial infarction. J Electrocardiol. 2002;35 Suppl:35-9.

[9] Pahlm O, Pettersson J, Thulin A, Feldman CL, Feild DQ, Wagner GS. Comparison of waveforms in conventional 12-lead ECGs and those derived from EASI leads in children. J Electrocardiol. 2003 Jan;36(1):25-31.

[10] Martínez JP, Almeida R, Olmos S, Rocha AP, and Laguna P. A Wavelet-Based ECG Delineator: Evaluation on Standard Database. IEEE Trans. Biomed. Eng., 2004; 51(4):570-581.

[11] Laguna P, Mark RG, Goldberger A and Moody GB. A Database for Evaluation of Algorithms for Measurement of QT and Other Waveform Intervals in the ECG. In Proc. Computers in Cardiology 1997, vol. 24. 673-6. IEEE Computer Society Press.

[12] Feild DQ, Feldman CL, Horacek BM, Improved EASI coefficients: Their Derivation, Values, and Performance, J. Electrocardiol., 2002;35, 23-33.

[13] Laguna P, Jané R, Caminal P. Automatic detection of wave boundaries in Multilead ECG signals: validation with the CSE database. Comput. Biomed. Res. 1994; 27:45-60

[14] The CSE Working Party. Recommendations for measurement standards in quantitative electrocardiography. Eur. Heart J. 1985;6:815-825.

[15] Almeida R, Martínez JP, Rocha AP, Olmos S, Laguna P. Improved QT Variability Quantification by Multilead Automatic Delineation. In Proc. Computers in Cardiology. 2005;32:503-506. IEEE Press. 